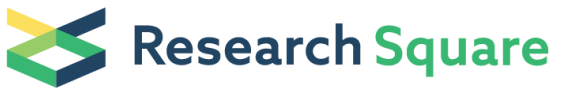 \\ Preprints are preliminary reports that have not undergone peer review. \\ They should not be considered conclusive, used to inform clinical practice, or referenced by the media as validated information.
}

\section{Clinical Outcomes of Hospitalized COVID-19 Patients with Renal Injury: A multi-hospital observational study from Wuhan}

\section{Kehong Chen}

Department of Nephrology, Daping Hospital, Army Medical University, Chongqing, China;

Yu Lei

Department of Infectious Disease, Huo Shen Shan Hospital, Wuhan, China

Yani He

Department of Nephrology, Daping Hospital, Army Medical University, Chongqing, China;

\section{Fei Xiao}

Department of Nephrology, Daping Hospital, Army Medical University, Chongqing, China;

\section{Yan Yu}

Department of Critical Care Medicine, Taikang Tongji Hospital, Wuhan, China;

\section{Xiaodong Lai}

Department of Critical Care Medicine, The People's Hospital of Wuhan University, Wuhan, China;

\section{Yang Liu}

Department of Infectious Disease, Taikang Tongji Hospital, Wuhan, China;

\section{Jiang Wang}

Department of Cardiology, Xinqiao Hospital, Army Medical University, Chongqing, China;

\section{Huanzi Dai ( $\nabla$ dhz084426@163.com )}

Department of Nephrology, Daping Hospital, Army Medical University, Chongqing, China;

\section{Research Article}

Keywords: COVID-19, disease progression, in-hospital prognosis, estimated glomerular filtration rate, acute kidney injury

Posted Date: February 26th, 2021

DOI: https://doi.org/10.21203/rs.3.rs-239498/v1

License: (9) (1) This work is licensed under a Creative Commons Attribution 4.0 International License. Read Full License

Version of Record: A version of this preprint was published at Scientific Reports on July 26th, 2021. See the published version at https://doi.org/10.1038/s41598-021-94570-1. 


\section{Abstract}

Renal injury is common in patients with coronavirus disease 2019 (COVID-19). We aimed to determine the relationship of estimated glomerular filtration rate (eGFR) and acute kidney injury (AKI) with the characteristics, progression, and prognosis of COVID-19 in-patients. We retrospectively reviewed 1851 COVID-19 patients admitted to 3 hospitals in Wuhan, China. Clinical, laboratory, radiological, treatment, complication, and outcome data were analyzed. Patients were stratified according to tertiles of eGFR ( $\geq 90$ vs. $60-89$ vs. $<60 \mathrm{~mL} / \mathrm{min} / 1.73 \mathrm{~m}^{2}$ ). The risk of reaching the composite endpoint-intensive care unit admission, invasive ventilation, or death-was compared. On admission, 25.5\% patients had renal impairment (eGFR $<90$ $\mathrm{mL} / \mathrm{min} / 1.73 \mathrm{~m}^{2}$ ), but only $2.6 \%$ patients had chronic kidney disease (CKD). The overall in-hospital AKI incidence was $6.7 \%$. Severe illness and comorbidities (hypertension, diabetes, CKD, and cardiovascular/cerebrovascular diseases) were more common among patients with low eGFR $\left(<90 \mathrm{~mL} / \mathrm{min} / 1.73 \mathrm{~m}^{2}\right)$. Despite the more frequent use of intensive oxygen therapy, continuous blood purification, and glucocorticoid treatment, the prognosis of these patients was unsatisfactory, with the incidence of the composite endpoint $(15.4 \%$ vs. $19.6 \%$ vs. $54.5 \% ; \mathrm{P}=0.000)$ and complications (AKI, respiratory failure, cardiac injury, coagulation disorders, sepsis, etc.) increasing with decreasing eGFR. Kaplan-Meier survival analysis revealed that patients with eGFR $<90 \mathrm{~mL} / \mathrm{min} / 1.73 \mathrm{~m}^{2}$ or AKI had significantly escalated risks of reaching the composite endpoint. Multivariate regression analysis showed that renal insufficiency (eGFR $<60 \mathrm{~mL} / \mathrm{min} / 1.73 \mathrm{~m}^{2}$ ) on admission and in-hospital AKI independently predicted poor prognosis among COVID-19 in-patients. Early and continuous renal-function monitoring and early AKI diagnosis are necessary to predict and prevent the progression of COVID-19.

\section{Introduction}

Coronavirus disease 2019 (COVID-19) is a pandemic viral disease caused by severe acute respiratory syndrome coronavirus 2 (SARS-CoV-2), which broke out in Wuhan, China, in December 2019 and spread worldwide ${ }^{1}$. As of November 7, 2020, there have been 49,247,980 confirmed COVID-19 cases and 1,241,981 deaths worldwide (announced by World Health Organization [WHO]). Infection with SARS-CoV-2 not only leads to severe acute respiratory syndrome but also causes damage to the kidneys $^{2}$, heart ${ }^{3}$, and other organs. A single-center study reported that $13.1-14.4 \%$ of COVID-19 patients had some renal injury markers on admission, including proteinuria, hematuria, and elevated serum creatinine (SCr) and blood urea nitrogen (BUN); furthermore, patients with markers of kidney impairment were at a higher risk for in-hospital death ${ }^{4}$. Unlike BUN or SCr alone, the estimated glomerular filtration rate (eGFR) reflects renal function more objectively and accurately, as it can be adjusted for age and gender. Thus, it is important to determine the relationship of the baseline renal function on admission with the characteristics, progression, and prognosis of COVID-19 patients.

Acute kidney injury (AKI) is an important complication of COVID-19, occurring in $0.5-9 \%$ of all cases and $10-30 \%$ of intensive care unit (ICU) or critically ill patients ${ }^{5-9}$. Although it has been reported that SARS-CoV-2 infection might not result in AKI or aggravate chronic kidney disease $(C K D)^{10}$, a retrospective analysis of $35 \mathrm{AKI}$ patients found that AKI at an early stage might serve as a negative prognostic indicator for COVID-19 ${ }^{11}$. Therefore, the clinical significance of AKI in patients infected with SARS-CoV-2 needs to be clarified.

In this study, we retrospectively analyzed an outbreak of COVID-19 in 3 hospitals in Wuhan, China.

The epidemiological, clinical, laboratory, and radiological characteristics as well as the treatment, complication, and outcome data of 1851 COVID-19 patients were stratified according to the eGFR on admission and reviewed.

We analyzed the association of both eGFR and AKI with patient prognosis. We expect our findings will shed light on the appropriate management of COVID-19 patients with renal impairment.

\section{Results}

\section{Patient characteristics}


A total of 1851 patients hospitalized with confirmed COVID-19 from 3 hospitals (1412 patients from Taikang Tongji Hospital, 335 patients from Huo Shen Shan Hospital, and 104 patients from Renmin Hospital of Wuhan University) were included in this study. To elucidate the clinical implications of renal dysfunction in COVID-19 patients, we stratified the patients into three groups according to their baseline eGFR as follows: 1379 patients $(74.5 \%), \geq 90 \mathrm{~mL} / \mathrm{min} / 1.73 \mathrm{~m}^{2} ; 360$ patients (19.4\%), 60-89 $\mathrm{mL} / \mathrm{min} / 1.73 \mathrm{~m}^{2}$; and 112 patients $(6.1 \%), \otimes 60 \mathrm{~mL} / \mathrm{min} / 1.73 \mathrm{~m}^{2}$. The characteristics of the 1851 COVID-19 patients are presented in Table 1. The median age was 62 years (IQR, 51-70 years), and 889 (48.0\%) patients were male. Severe cases accounted for $37.9 \%$ of the study population. The most common symptoms on admission were fever (1099 patients, $59.3 \%)$ and cough (1044 patients, $56.4 \%$ ). Asymptomatic patients accounted for $2.1 \%$ of the study population. The median duration from symptom onset to admission was 25 days (IQR, 14-38 days). Comorbidities were present in half the patients (50.5\%), with hypertension (697 patients, $37.7 \%$ ) and diabetes (310 patients, $16.8 \%$ ) being the most prevalent comorbidities. Only $2.6 \%$ patients had a history of CKD. 
Table 1

Clinical characteristics of COVID-19 patients stratified by eGFR tertiles

\begin{tabular}{|c|c|c|c|c|c|}
\hline \multirow[t]{3}{*}{ Variable } & \multirow{3}{*}{$\begin{array}{l}\text { All patients } \\
(\mathrm{N}=1851)\end{array}$} & \multicolumn{3}{|c|}{ eGFR, $\mathrm{ml} / \mathrm{min} / 1.73 \mathrm{~m}^{2}$} & \multirow{3}{*}{$\begin{array}{l}\mathrm{P} \\
\text { value }^{\mathrm{a}}\end{array}$} \\
\hline & & $\geq 90$ & $60-89$ & 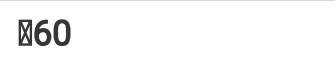 & \\
\hline & & $(N=1379)$ & $(\mathrm{N}=360)$ & $(N=112)$ & \\
\hline $\mathbf{N}(\%)$ & 1851 & $1379(74.5)$ & $360(19.4)$ & $112(6.1)$ & \\
\hline Age (years) & $62(51,70)$ & $59(48,67)$ & $73(63,81)$ & $72(64,81)$ & 0.001 \\
\hline Male sex, n/N (\%) & $889 / 1851(48.0)$ & $650 / 1379(47.1)$ & $180 / 360(50.0)$ & $59 / 112(52.6)$ & 0.373 \\
\hline $\mathrm{BMI}\left(\mathrm{kg} / \mathrm{m}^{2}\right)$ & $23.42(21.25,25.51)$ & 23.32(21.22,25.39) & $23.56(21.22,26.03)$ & $23.71(22.03,25.83)$ & 0.579 \\
\hline $\begin{array}{l}\text { Systolic pressure on } \\
\text { admission }(\mathrm{mm} \mathrm{Hg})\end{array}$ & $130(120,142)$ & $130(120,141)$ & $132(120,145)$ & $131.5(120,145)$ & 0.000 \\
\hline $\begin{array}{l}\text { Diastolic pressure on } \\
\text { admission }(\mathrm{mm} \mathrm{Hg})\end{array}$ & $80(74,88)$ & $80(73.5,88)$ & $80(74,88)$ & $80(75,90)$ & 0.183 \\
\hline $\begin{array}{l}\text { Mean arterial pressure } \\
\text { on admission ( } \mathrm{mm} \\
\mathrm{Hg} \text { ) }\end{array}$ & $96.67(89.33,106)$ & $96.66(90,104.66)$ & $98.5(91.66,107.33)$ & $95.33(88.08,105.33)$ & 0.008 \\
\hline \multicolumn{6}{|l|}{$\begin{array}{l}\text { Disease severity, } \mathrm{n} / \mathrm{N} \\
\text { (\%) }\end{array}$} \\
\hline Non-severe & 1149/1851(62.07) & $930 / 1379(67.44)$ & $188 / 360(52.22)$ & $31 / 112(27.67)$ & 0.000 \\
\hline Severe & 700/1848(37.87) & 447/1379(32.41) & $172 / 360(47.78)$ & $81 / 112(72.32)$ & 0.000 \\
\hline $\begin{array}{l}\text { Time from symptom } \\
\text { onset to admission } \\
\text { (days) }\end{array}$ & $25(14,38)$ & $26(14,39)$ & $21(14,36)$ & $17(8,36)$ & 0.017 \\
\hline \multicolumn{6}{|l|}{$\begin{array}{l}\text { Symptoms at onset, } \\
\mathrm{n} / \mathrm{N}(\%)\end{array}$} \\
\hline Fever & 1099/1851(59.37) & $838 / 1379(60.76)$ & $208 / 360(57.77)$ & $53 / 112(47.32)$ & 0.001 \\
\hline Cough & $1044 / 1851(56.40)$ & 794/1379(57.57) & $196 / 360(54.44)$ & $54 / 112(48.21)$ & 0.112 \\
\hline Sputum production & $309 / 1851(16.69)$ & 248/1379(17.98) & 48/360(13.33) & $13 / 112(11.60)$ & 0.036 \\
\hline Fatigue & 766/1851(41.38) & 550/1379(39.88) & $166 / 360(46.11)$ & $50 / 112(44.64)$ & 0.080 \\
\hline Shortness of breath & 491/1851(26.52) & $343 / 1379(24.87)$ & $108 / 360(30.00)$ & 40/112(35.71) & 0.011 \\
\hline Chills & 253/1851(13.67) & 181/1379(13.12) & $53 / 360(13.72)$ & 19/112(16.96) & 0.424 \\
\hline Hemoptysis & $100 / 1851(5.43)$ & 77/1379(5.58) & $18 / 360(5)$ & $5 / 112(4.46)$ & 0.821 \\
\hline Headache/dizziness & $88 / 1851(4.75)$ & 65/1379(4.71) & $19 / 360(5.28)$ & 4/112(3.57) & 0.791 \\
\hline $\begin{array}{l}\text { Nasal } \\
\text { congestion/rhinorrhea }\end{array}$ & 113/1851(6.10) & 88/1379(6.38) & $21 / 360(5.83)$ & 4/112(3.57) & 0.784 \\
\hline Pharyngodynia & 236/1851(12.75) & 179/1379(12.98) & $45 / 360(12.5)$ & 12/112(10.71) & 0.778 \\
\hline Myalgia/arthralgia & 192/1851(10.37) & 136/1379(9.86) & $42 / 360(11.66)$ & $14 / 112(12.5)$ & 0.455 \\
\hline
\end{tabular}

Data are expressed as median (interquartile range) or no./total no. (\%). ${ }^{\text {ap }}$ values were calculated using the Kruskal-Wallis test or the chi-square test.

COVID-19, coronavirus disease 2019; eGFR, estimated glomerular filtration rate; BMI, body mass index 


\begin{tabular}{|c|c|c|c|c|c|}
\hline \multirow[t]{3}{*}{ Variable } & \multirow{3}{*}{$\begin{array}{l}\text { All patients } \\
(\mathrm{N}=1851)\end{array}$} & \multicolumn{3}{|c|}{ eGFR, $\mathrm{ml} / \mathrm{min} / 1.73 \mathrm{~m}^{2}$} & \multirow{3}{*}{$\begin{array}{l}P \\
\text { value }^{a}\end{array}$} \\
\hline & & $\geq 90$ & $60-89$ & 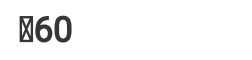 & \\
\hline & & $(N=1379)$ & $(\mathrm{N}=360)$ & $(N=112)$ & \\
\hline Chest pain & 43/1851(2.32) & 33/1379(2.39) & $8 / 360(2.22)$ & 2/112(1.78) & 1.000 \\
\hline $\begin{array}{l}\text { Chest } \\
\text { distress/palpitations }\end{array}$ & $371 / 1851(20.04)$ & 257/1379 (18.64) & $90 / 360(25.00)$ & $24 / 112(21.43)$ & 0.025 \\
\hline Anorexia & 238/1851(12.85) & 158/1379(11.45) & 66/360(18.33) & 14/112(12.5) & 0.003 \\
\hline Diarrhea & 224/1851(12.11) & 165/1379(11.97) & $50 / 360(13.89)$ & 9/112(8.04) & 0.241 \\
\hline Nausea/vomiting & $56 / 1851(3.02)$ & 40/1379(2.90) & 13/360(3.61) & $3 / 112(2.67)$ & 0.708 \\
\hline $\begin{array}{l}\text { Disturbance of } \\
\text { consciousness }\end{array}$ & $8 / 1851(0.43)$ & $7 / 1379(0.51)$ & $0 / 360(0)$ & 1/112(0.89) & 0.230 \\
\hline Asymptomatic & $38 / 1851(2.05)$ & $32 / 1379(2.32)$ & $4 / 360(1.11)$ & 2/112(1.79) & 0.039 \\
\hline \multicolumn{6}{|l|}{$\begin{array}{l}\text { Comorbidities, } \mathrm{n} / \mathrm{N} \\
(\%)\end{array}$} \\
\hline Any & $934 / 1851(50.45)$ & $598 / 1379(43.36)$ & 238/360(66.11) & $98 / 112(87.5)$ & 0.000 \\
\hline Hypertension & 697/1850(37.67) & 424/1379(30.75) & 191/360(53.05) & $82 / 112(73.21)$ & 0.000 \\
\hline Diabetes & 310/1848(16.77) & 200/1379(14.50) & $73 / 360(20.27)$ & $37 / 112(33.03)$ & 0.000 \\
\hline $\begin{array}{l}\text { Coronary heart } \\
\text { disease }\end{array}$ & 199/1850(10.75) & 104/1379(7.54) & $62 / 360(17.22)$ & $33 / 112(29.46)$ & 0.000 \\
\hline $\begin{array}{l}\text { Chronic obstructive } \\
\text { pulmonary disease }\end{array}$ & 78/1847(4.22) & 53/1379(3.84) & $18 / 360(5.00)$ & $7 / 112(6.25)$ & 0.342 \\
\hline $\begin{array}{l}\text { Cerebrovascular } \\
\text { disease }\end{array}$ & 129/1849(6.97) & 71/1379(5.15) & $41 / 360(11.38)$ & 17/112(15.17) & 0.000 \\
\hline $\begin{array}{l}\text { Chronic kidney } \\
\text { disease }\end{array}$ & 48/1849(2.59) & $6 / 1379(0.44)$ & $8 / 360(2.22)$ & $34 / 112(30.35)$ & 0.000 \\
\hline Hepatitis B infection & 27/1848(1.46) & 18/1379(1.31) & $6 / 360(1.67)$ & $3 / 112(2.67)$ & 0.512 \\
\hline Cancer & $38 / 1849(2.05)$ & 29/1379(2.10) & 5/360(1.38) & 4/112(3.57) & 0.347 \\
\hline \multicolumn{6}{|c|}{$\begin{array}{l}\text { Data are expressed as median (interquartile range) or no./total no. (\%). }{ }^{\text {ap }} \text { values were calculated using the Kruskal-Wallis } \\
\text { test or the chi-square test. }\end{array}$} \\
\hline \multicolumn{6}{|c|}{ COVID-19, coronavirus disease 2019; eGFR, estimated glomerular filtration rate; BMI, body mass index } \\
\hline
\end{tabular}

Compared with patients in the highest tertile of eGFR ( $\left.\geq 90 \mathrm{~mL} / \mathrm{min} / 1.73 \mathrm{~m}^{2}\right)$, patients in the lower 2 tertiles were older, had a higher prevalence of severe illness, were more likely to experience shortness of breath and chest distress/palpitations, and were less likely to have fever and sputum production. Moreover, comorbidities, including hypertension, diabetes, coronary heart disease, cerebrovascular disease, and CKD, were present more often among patients in the lower 2 tertiles.

\section{Laboratory and radiological findings}

Table 2 shows the laboratory and radiological findings on admission. In the overall study population, the median values of all laboratory indicators were within the normal range. Of the 1463 CT scans that were performed on admission, $87.6 \%$ revealed abnormal results.

Table 2. Laboratory and radiological findings of COVID-19 patients stratified according to eGFR tertiles 


\begin{tabular}{|c|c|c|c|c|c|}
\hline \multirow[t]{2}{*}{ ariable } & \multirow{2}{*}{$\begin{array}{l}\text { All patients } \\
(\mathrm{N}=1851)\end{array}$} & \multicolumn{3}{|c|}{ eGFR, $\mathrm{ml} / \min / 1.73 \mathrm{~m}^{2}$} & \multirow{2}{*}{$\begin{array}{c}P \\
\text { value }\end{array}$} \\
\hline & & $\begin{array}{c}\geq 90 \\
(\mathrm{~N}=1379)\end{array}$ & $\begin{array}{c}60-89 \\
(\mathrm{~N}=360)\end{array}$ & $\begin{array}{c}\square 60 \\
(N=112)\end{array}$ & \\
\hline \multicolumn{6}{|l|}{$\begin{array}{l}\text { aboratory } \\
\text { ndings at } \\
\text { Imission }\end{array}$} \\
\hline ematological & & & & & \\
\hline $\begin{array}{l}\text { zukocyte Count, } \\
10^{9} / \mathrm{L}\end{array}$ & $5.84(4.74,7.13)$ & $5.75(4.7,7.0)$ & $6(4.8,7.22 \square$ & $6.715 \square 5.52,9.32 \square$ & 0.060 \\
\hline 3.5- n/N (\%) & $95 / 1841(5.16)$ & 68/1370ロ4.96ロ & 23/359a6.40ם & 4/112₫3.57ロ & 0.412 \\
\hline $.5-\mathrm{n} / \mathrm{N}(\%)$ & $169 / 1841011.26 \square$ & 103/137007.51ם & $40 / 359 \square 11.14 \square$ & 26/112ロ23.21ם & 0.000 \\
\hline $\begin{array}{l}\text { mphocyte } \\
\text { sunt, } \times 10^{9} / \mathrm{L}\end{array}$ & $1.53 \square 1.12,2 \square$ & $1.6(1.2,2.07)$ & $1.33 \square 0.99,1.77 \square$ & $1.12 \llbracket 0.62,1.79 \square$ & 0.000 \\
\hline $1.1-\mathrm{n} / \mathrm{N}(\%)$ & 433/1841ロ23.510 & 264/1370ロ19.27ロ & $114 / 359 \square 31.75 \square$ & $55 / 112 \square 49.10 \square$ & 0.000 \\
\hline $\begin{array}{l}\text { atelet Count, } \\
10^{9} / \mathrm{L}\end{array}$ & $216 \square 176,265 \square$ & $220(183,270)$ & $209 \square 165,257 \square$ & 196ロ144,237ロ & 0.000 \\
\hline 125- n/N (\%) & 147/1839ロ7.99ロ & 88/1368ロ6.43ロ & $32 / 359 \square 8.91 \square$ & $27 / 112 \square 24.10 \square$ & 0.000 \\
\hline $50-\mathrm{n} / \mathbf{N}(\%)$ & 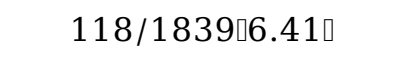 & 89/1368ロ6.50ロ & $24 / 359 \square 6.68 \square$ & $5 / 112 \llbracket 4.46 \square$ & 0.685 \\
\hline $\begin{array}{l}\text { emoglobin, } \mathrm{g} / \mathrm{L} \\
\text { iochemical }\end{array}$ & $120 \square 109,132 \square$ & $121(111,133)$ & $117 \square 105,129 \square$ & $109(89,124)$ & 0.000 \\
\hline $\begin{array}{l}\text { reatinine, } \\
\mathrm{mol} / \mathrm{L}\end{array}$ & $56.34(46.28,69.53)$ & $51.4(43.21,60.31)$ & 73.61ם65.3,84.7ロ & $126.88 \square 97.42,181.7 \square$ & 0.000 \\
\hline $04-\mathrm{n} / \mathrm{N}$ (\%) & 87/1851₫4.70ロ & $0 / 1379 \square 0 \square$ & $9 / 360 \square 2.5 \square$ & $78 / 112 \square 69.64 \square$ & 0.000 \\
\hline $\begin{array}{l}\text { zak creatinine, } \\
\mathrm{mol} / \mathrm{L}\end{array}$ & $59.99(49.54,74.83)$ & $55.23(47,64.87)$ & $77.75(67.14,89.68)$ & $145.6 \square 106.59,212.03 \square$ & 0.000 \\
\hline $\begin{array}{l}\text { rea nitrogen, } \\
\mathrm{mol} / \mathrm{L}\end{array}$ & $4.9 \square 4.03,6.1 \square$ & $4.61(3.84,5.57)$ & $5.71 \square 4.6,7.17 \square$ & $11.025 \square 7.76,15.40 \square$ & 0.000 \\
\hline $.2-\mathrm{n} / \mathrm{N}(\%)$ & 249/1851ロ13.45ם & 73/1379ם5.29ם & $86 / 360 \llbracket 23.88 \square$ & $90 / 112 \square 80.35 \square$ & 0.000 \\
\hline lbumin, $g / L$ & $37.73(34.26,40.65)$ & $38.3(35.1,41.03)$ & $36.22(32.16,39.47)$ & $33.88 \square 30.05,37.66 \square$ & 0.000 \\
\hline $35-\mathrm{n} / \mathrm{N}(\%)$ & $547 / 1845 \square 29.64 \square$ & $334 / 1374024.30 \square$ & $150 / 360 \square 41.66 \square$ & 63/111056.75ם & 0.000 \\
\hline $\begin{array}{l}\text { Jtal bilirubin, } \\
\mathrm{mol} / \mathrm{L}\end{array}$ & $10.62 \square 8.32,13.71 \square$ & $10.58(8.32,13.37)$ & $10.78(8.32,14.94 \square$ & 10.58凸8.28,15.87ロ & 0.256 \\
\hline $0.5-\mathrm{n} / \mathrm{N}(\%)$ & $113 / 1844 \llbracket 6.12 \square$ & 65/1374ロ4.73ロ & $32 / 360 \square 8.88 \square$ & $16 / 110 \square 14.54 \square$ & 0.000 \\
\hline $\begin{array}{l}\text { lanine } \\
\text { ninotransferase, } \\
/ \mathrm{L}\end{array}$ & $21.81 \square 14.58,36.7 \square$ & $18.83 \square 11.65,30.6 \square$ & $21.11(14.27,32.96)$ & $22.64(14.86,38.05)$ & 0.000 \\
\hline $5-\mathbf{n} / \mathbf{N}(\%)$ & $333 / 1844018.05 \square$ & 256/1374018.63ם & $59 / 360016.38 \square$ & 18/110ø16.36ロ & 0.549 \\
\hline $\begin{array}{l}\text { spartate } \\
\text { ninotransferase, } \\
/ \mathrm{L}\end{array}$ & $20.03(15.08,28.81)$ & $19.42(14.49,27.65)$ & $21.41(16.97,31.28)$ & $24.4 \llbracket 17.65,40.79 \square$ & 0.000 \\
\hline $0-\mathbf{n} / \mathbf{N}(\%)$ & 181/1425012.70ロ & $112 / 1046 \square 10.70 \square$ & $44 / 284 \llbracket 15.49 \square$ & 25/95026.31ם & 0.000 \\
\hline $\begin{array}{l}\text { actate } \\
\text { əhydrogenase, } \\
\text { /L }\end{array}$ & $178.45(149.28,221.01)$ & $173.58(145.11,212.06)$ & $194.1(161.93,234.81)$ & )218.5ロ164.81,367.06ロ & 0.008 \\
\hline 71-n/N (\%) & 251/1851ロ13.56ロ & 147/1379ロ10.65ロ & 63/360ロ17.5ロ & $41 / 112 \llbracket 36.60 \square$ & 0.000 \\
\hline $\begin{array}{l}\text { reatine kinase- } \\
\mathrm{B}, \mathrm{U} / \mathrm{L}\end{array}$ & $8.15 \square 6.2,10.87 \square$ & $8.04(6.3,10.76)$ & $8.37 \square 6.11,10.78 \square$ & $8.48 \square 5.87,11.2 \square$ & 0.528 \\
\hline 4- n/N (\%) & $32 / 1232 \llbracket 2.59 \square$ & $21 / 945 \square 2.22 \square$ & $4 / 226 \square 1.76 \square$ & 7/61ロ11.47ロ & 0.001 \\
\hline yoglobin, & 21₫13.2,33.33 & $21(11.4,26.56)$ & $29.16 \square 21,47.87 \square$ & $53.32 \llbracket 29.31,253.44 \square$ & 0.000 \\
\hline
\end{tabular}


$y / \mathrm{mL}$

\section{8- n/N (\%) \\ igh-sensitivity irdiac Troponin $\mathrm{ng} / \mathrm{mL}$}

\section{.04- n/N (\%)}

215/1015ロ21.18ロ

fection-related dices

-reactive cotein, $\mathrm{mg} / \mathrm{L}$

$0-\mathbf{n} / \mathbf{N}$ (\%)

iterleukin-6,

$\mathrm{y} / \mathrm{mL}$

\section{- n/N (\%)}

ocalcitonin,

$\mathrm{y} / \mathrm{mL}$

0.05- n/N (\%)

0.5- $\mathbf{n} / \mathbf{N}$ (\%)

oagulation

nction

othrombin

me, $\mathrm{s}$

3.4- n/N (\%)

2.5- n/N (\%)

ctivated partial romboplastin

me, $\mathrm{s}$

25.1- n/N (\%)

6.5- n/N (\%)

ibrinogen,

$\mathrm{g} / \mathrm{dL}$

238- n/N (\%)

98- n/N (\%)

-dimer, $\mathrm{ng} / \mathrm{mL}$

500- n/N (\%)

rinalysis

oteinuria- $\mathbf{n} / \mathbf{N}$

6)

$\begin{array}{lc}\text { Negative } & 989 / 1223 \square 80.86 \square \\ \text { Positive } & 234 / 1223 \square 19.13 \square \\ + & 211 / 1223 \square 17.25 \square \\ ++\sim+++ & 23 / 1223 \square 1.88 \square\end{array}$

ematuria- $\mathbf{n} / \mathbf{N}$

6)

$\begin{array}{lc}\text { Negative } & 847 / 1219 \square 69.48 \square \\ \text { Positive } & 372 / 1219 \square 30.52 \square \\ + & 307 / 1219 \square 25.18 \square \\ ++\sim+++ & 65 / 1219 \square 5.33 \square\end{array}$

$0.79 \llbracket 0.5,8.18 \square$

399/1743ロ22.89■

2.99ロ1.5,8.01凸

403/1499ロ26.88ロ

$0.05 \square 0.03,0.08 \square$

618/1368ロ45.17ロ

71/1368(5.19)

$12.22 \square 11.4,13.1 \square$

0

$560 / 1339 \square 41.82 \square$

$30.2 \square 27.6,33 \square$

89/1338ロ6.65ロ

137/1338ロ10.23ロ

$276 \square 217,336 \square$

372/1336ロ27.84ロ

40/1336ロ2.99ロ

63ロ2,304ロ

185/932ロ19.84ロ

$$
\begin{aligned}
& 47 / 741 \square 6.34 \square \\
& 0.01(0,0.02)
\end{aligned}
$$

37/199ロ18.59

$0.03(0.01,0.069)$

104/754₫13.79ロ

77/203ロ37.93ロ
786/916ロ85.80ロ

130/916ロ14.19!

124/916ロ13.53ロ

6/916ロ0.65ロ

$1.69 \square 0.5,11.28 \square$

$92 / 346 \square 26.58 \square$

$6.18 \square 2.38,13.89 \square$

127/289ロ43.94ロ

$0.06 \square 0.04,0.12 \square$

164/261ロ62.83ロ

16/261(6.13)

12.6ロ11.7,13.5凸

0

$141 / 276 \square 51.08 \square$

$30.36 \square 28.08,33.03 \square$

$16 / 276 \square 5.79 \square$

$25 / 276 \square 9.05 \square$

$302 \square 245,346 \square$

54/276ロ19.56ロ

$8 / 276 \square 2.89$ 凸

$155.5 \square 60,451.5 \square$

$45 / 201 \square 22.38 \square$

$175 / 240 \square 72.91 \square$

$65 / 240 \square 27.08 \square$

$59 / 240 \square 24.58 \square$

$6 / 240 \square 2.5 \square$

$162 / 239 \square 67.78 \square$
$77 / 239 \square 32.22 \square$
$63 / 239 \square 26.35 \square$
$14 / 239 \square 5.85 \square$

$162 / 239 \square 67.78 \square$

$261 / 913 \square 28.59 \square$

$223 / 913 \square 24.42 \square$

38/913ロ4.16ロ
29/61ロ47.54ロ

0.000

$0.058(0.016,0.149) 0.000$

$34 / 58 \square 58.62 \square \quad 0.000$

$10.9(0.74,72.75 \square \quad 0.000$

$55 / 109 \square 50.45 \square \quad 0.000$

$10.94 \llbracket 4.94,36.63 \square \quad 0.000$

50/82ロ60.97ロ 0.000

$0.16 \square 0.07,0.57 \square \quad 0.030$

78/96ロ81.25ロ 0.000

26/96(27.08) $\quad 0.000$

$13.07 \square 12,14.825 \square \quad 0.000$

0

$56 / 90 \square 62.22 \square \quad 0.000$

$31.34 \square 28.83,34.58 \square \quad 0.175$

$8 / 90 \square 8.88 \square \quad 0.589$

17/90ロ18.88ロ 0.018

$306 \square 251,390 \square \quad 0.000$

$22 / 89 \square 24.71 \square \quad 0.000$

$3 / 89 \square 3.37 \square \quad 0.922$

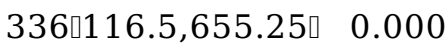

$27 / 72 \llbracket 37.5 \square$

0.000

$\begin{array}{ll}28 / 67 \square 41.79 \square & 0.000 \\ 39 / 67 \square 58.21 \square & 0.000 \\ 28 / 67 \square 41.79 \square & 0.000 \\ 11 / 67 \square 16.41 \square & 0.000\end{array}$

33/67ロ49.25ロ

0.001

$34 / 67 \square 50.75 \square$

0.001

$21 / 67 \square 31.34 \square$

0.409

13/67ロ19.40ロ

0.000 


\section{hest computed \\ imography \\ ndings-- $\mathbf{n} / \mathbf{N}$ \\ o)}

bnormalities on

rest CT

Sub-pleura

Unilateral

Bilateral 1282/1463ø87.63ロ

117/1463ロ8.00ロ

129/1463ロ8.82ロ

1036/1463ロ70.81凸
942/1079ロ87.30ロ

102/1079ø6.97ロ

107/1079घ7.31ם

733/1079ロ67.93ロ
273/304ロ89.80ロ

$12 / 304 \square 3.95 \square$

19/304ロ6.25ם

242/304ロ79.61ロ
67/80ロ83.75ロ

0.030

3/80ロ3.75ロ

0.003

3/80ロ3.75ロ

0.036

$61 / 80 \square 76.25 \square$

0.000

Data are expressed as median (interquartile range) or no./total no. (\%). ${ }^{\text {ap }}$ values were calculated using the Kruskal-Wallis test or chisquare test.

COVID-19, coronavirus disease 2019; eGFR, estimated glomerular filtration rate

Compared with patients in the highest eGFR tertile, patients in the lower 2 tertiles showed significantly elevated cardiac injury indicators (high-sensitivity cardiac troponin I, myoglobin, and lactate dehydrogenase), liver injury indicators (alanine and aspartate aminotransferases), inflammation-related indicators (C-reactive protein, interleukin-6, and procalcitonin), and coagulation function indicators (prothrombin time, fibrinogen, and d-dimer), and significantly decreased platelet count, hemoglobin, and albumin. Unlike patients in the highest eGFR tertile, patients in the lower 2 tertiles tended to have bilateral pneumonia rather than subpleural or unilateral pneumonia.

\section{Treatment, complications, and clinical outcomes}

The treatment, complications, and in-hospital clinical outcomes of the patients are summarized in Table 3. Of the 1851 patients, 58.9\% patients required oxygen support in the hospital. The most intense level was recorded, including low-flow (46.7\%) and high-flow nasal cannula (HFNC, 6.2\%) oxygen inhalation, noninvasive mechanical ventilation (NMV, 1.9\%), and invasive mechanical ventilation (IMV, 4.1\%). Only 1.1\% patients required continuous renal replacement therapy (CRRT). Extracorporeal membrane oxygenation was used in 2 patients. Antiviral therapy was the most common treatment (64.7\%), followed by antibiotic therapy (41.4\%) and glucocorticoids (11.9\%). Renin-angiotensin system (RAS) inhibitors and diuretics were administered to $10.9 \%$ and $10.2 \%$ patients, respectively. In addition, 23 (1.3\%), 44 (2.5\%), and 16 (0.9\%) patients were treated with tocilizumab, umbilical cord mesenchymal stem cells, and convalescent plasma, respectively. Coagulation disorder (66.2\%) was the most frequent complication, followed by anemia, hypoproteinemia, electrolyte disturbances, acute cardiac injury, respiratory failure, acidosis, and ARDS. A total of 1690 patients were followed up for a median of 16 days, during which 90.7\% (1532) patients were discharged, 4.9\% (83) patients died, and 4.4\% (75) patients remained hospitalized. The remaining patients (161) were lost to follow-up. 
Table 3

Treatment, complications, and clinical outcomes of COVID-19 patients stratified according to eGFR tertiles

\begin{tabular}{|c|c|c|c|c|c|}
\hline \multirow[t]{3}{*}{ Variable } & \multirow{3}{*}{$\begin{array}{l}\text { All patients } \\
(\mathrm{N}=1851)\end{array}$} & \multicolumn{3}{|c|}{ eGFR, $\mathrm{ml} / \mathrm{min} / 1.73 \mathrm{~m}^{2}$} & \multirow{3}{*}{$\begin{array}{l}\mathrm{P} \\
\text { value }^{\mathrm{a}}\end{array}$} \\
\hline & & $\geq 90$ & $60-89$ & $\llbracket 60$ & \\
\hline & & $(N=1379)$ & $(N=360)$ & $(N=112)$ & \\
\hline \multicolumn{6}{|l|}{ Treatment in hospital, $\mathrm{n} / \mathrm{N}$ (\%) } \\
\hline Oxygen therapy & $1091 / 1851(58.94)$ & $791 / 1379$ (57.36) & $\begin{array}{l}222 / 360 \\
(61.67)\end{array}$ & 78/112(69.64) & 0.020 \\
\hline Nasal duct/mask & $865 / 1851(46.73)$ & 659/1379(47.79) & $170 / 360(47.22)$ & $36 / 112(32.14)$ & 0.006 \\
\hline High-flow nasal cannula & $114 / 1851(6.16)$ & 76/1379(5.51) & $24 / 360(6.67)$ & $14 / 112(12.50)$ & 0.011 \\
\hline $\begin{array}{l}\text { Noninvasive mechanical } \\
\text { ventilation }\end{array}$ & 36/1851(1.94) & 20/1379(1.45) & $7 / 360(1.94)$ & $9 / 112(8.04)$ & 0.000 \\
\hline $\begin{array}{l}\text { Invasive mechanical } \\
\text { ventilation }\end{array}$ & 76/ 1851(4.11) & $36 / 1379(2.61)$ & $21 / 360(5.83)$ & 19/112(16.96) & 0.000 \\
\hline $\begin{array}{l}\text { Continuous renal replacement } \\
\text { therapy }\end{array}$ & 21/1733(1.21) & $7 / 1283(0.54)$ & $4 / 345(1.15)$ & 10/105(9.52) & 0.000 \\
\hline $\begin{array}{l}\text { Extracorporeal membrane } \\
\text { oxygenation }\end{array}$ & $2 / 1851(0.11)$ & $2 / 1379(0.15)$ & $0 / 0$ & $0 / 0$ & - \\
\hline Antibiotic therapy & 713/1733(41.14) & $500 / 1284(38.94)$ & $149 / 344(43.31)$ & $64 / 105(60.95)$ & 0.000 \\
\hline Antiviral therapy & $1121 / 1733(64.68)$ & $821 / 1283(63.99)$ & $225 / 345(65.21)$ & 75/105(71.42) & 0.301 \\
\hline Glucocorticoids & 204/1721(11.85) & $122 / 1274(9.57)$ & 45/343(13.11) & $37 / 104(35.57)$ & 0.000 \\
\hline RAS inhibitors ${ }^{b}$ & 188/1729(10.87) & $111 / 1281(8.66)$ & $54 / 343(15.74)$ & 23/105(21.90) & 0.000 \\
\hline Diuretics & 177/1731(10.22) & 91/1282(7.09) & $53 / 344(15.40)$ & 33/105(31.42) & 0.000 \\
\hline Tocilizumab & 23/1728(1.33) & 14/1279(1.09) & 6/344(1.74) & $3 / 105(2.85)$ & 0.169 \\
\hline $\begin{array}{l}\text { Umbilical cord mesenchymal } \\
\text { stem cells }\end{array}$ & 44/1728(2.54) & $32 / 1282(2.49)$ & $10 / 342(2.92)$ & 2/104(1.92) & 0.854 \\
\hline Convalescent plasma & 16/1731(0.92) & $7 / 1285(0.54)$ & $8 / 341(2.34)$ & 1/105(0.95) & 0.11 \\
\hline \multicolumn{6}{|l|}{ Complications, n/N (\%) } \\
\hline Any complication & 1372/1747(78.53) & 976/1293(75.48) & $296 / 348(85.06)$ & 100/106(94.34) & 0.000 \\
\hline Respiratory failure & 139/1742(7.98) & 71/1290(5.50) & $35 / 346(10.11)$ & 33/106(31.13) & 0.000 \\
\hline $\begin{array}{l}\text { Acute respiratory distress } \\
\text { syndrome }\end{array}$ & $80 / 1742(4.59)$ & 39/1290(3.02) & 20/346(5.78) & 21/106(19.81) & 0.000 \\
\hline
\end{tabular}

Data are expressed as no./total no. (\%).

ap values were calculated using the chi-square test.

${ }^{\text {b}}$ RAS inhibitors included angiotensin-converting-enzyme inhibitors and angiotensin receptor blockers. RAS, reninangiotensin system.

'The composite endpoint comprised admission to the intensive care unit or mechanical ventilation or death, whichever occurred first.

COVID-19, coronavirus disease 2019; eGFR, estimated glomerular filtration rate 


\begin{tabular}{|c|c|c|c|c|c|}
\hline \multirow[t]{3}{*}{ Variable } & \multirow{3}{*}{$\begin{array}{l}\text { All patients } \\
(\mathrm{N}=1851)\end{array}$} & \multicolumn{3}{|c|}{ eGFR, $\mathrm{ml} / \mathrm{min} / 1.73 \mathrm{~m}^{2}$} & \multirow{3}{*}{$\begin{array}{l}P \\
\text { value }^{a}\end{array}$} \\
\hline & & $\geq 90$ & $60-89$ & $\triangle 60$ & \\
\hline & & $(N=1379)$ & $(\mathrm{N}=360)$ & $(N=112)$ & \\
\hline Acute heart failure & $42 / 1742(2.41)$ & $14 / 1290(1.08)$ & 13/346(3.75) & 15/106(14.15) & 0.000 \\
\hline Acute cardiac injury & $300 / 1663(18.03)$ & 151/1239(12.18) & $98 / 326(30.06)$ & $51 / 98(52.04)$ & 0.000 \\
\hline Acute kidney injury & 114/1708(6.67) & $74 / 1266(5.85)$ & $23 / 340(6.76)$ & 18/102(17.65) & 0.000 \\
\hline Stage 1 & 70/1708(4.10) & $43 / 1266(3.40)$ & 13/340 (3.82) & 14/102(13.73) & 0.000 \\
\hline Stage 2 & 26/1708(1.52) & $20 / 1266(1.58)$ & 4/340 (1.18) & 2/102(1.96) & 0.806 \\
\hline Stage 3 & 18/1708(1.05) & $10 / 1266(0.79)$ & $6 / 340(1.76)$ & 2/102(1.96) & 0.039 \\
\hline Coagulation disorders & $906 / 1368(66.22)$ & 619/999(61.96) & $213 / 280(76.07)$ & 74/89(83.14) & 0.000 \\
\hline Sepsis & 18/1742(1.03) & 10/1290(0.77) & $3 / 346(0.86)$ & 5/106(4.71) & 0.006 \\
\hline Shock & $44 / 1742(2.52)$ & $17 / 1290(1.31)$ & 13/346(3.75) & 14/106(13.20) & 0.000 \\
\hline Septic shock & 29/1742(1.66) & 20/1290(1.55) & $9 / 346(2.60)$ & 0/106(0.00) & 1.000 \\
\hline Secondary infection & 18/1742(1.03) & $12 / 1290(0.93)$ & $3 / 346(0.86)$ & 3/106(2.83) & 0.168 \\
\hline Anemia & 600/1841(32.59) & $391 / 1370(28.54)$ & $147 / 359(40.95)$ & $62 / 112(55.36)$ & 0.000 \\
\hline Hypoproteinemia & 507/1743(29.08) & $304 / 1289(23.58)$ & $145 / 348(41.66)$ & $58 / 106(54.71)$ & 0.000 \\
\hline Electrolyte disturbances & 318/1695(18.76) & 199/1250(15.92) & $75 / 340(22.05)$ & $44 / 105(41.90)$ & 0.000 \\
\hline Acidosis & $81 / 1713(4.72)$ & $35 / 1268(2.76)$ & $22 / 341(6.45)$ & 24/104(23.07) & 0.000 \\
\hline \multicolumn{6}{|l|}{$\begin{array}{l}\text { Clinical outcome at data } \\
\text { cutoff, } \mathrm{n} / \mathrm{N}(\%)\end{array}$} \\
\hline Discharge from hospital & 1532/1690(90.65) & $1174 / 1253(93.70)$ & 299/336(88.98) & $59 / 101(58.41)$ & 0.000 \\
\hline Hospitalization & $75 / 1690(4.44)$ & 45/1253(3.59) & 14/336(4.16) & 16/101(15.84) & 0.000 \\
\hline Death & $83 / 1690(4.91)$ & $30 / 1253(2.38)$ & 25/336(7.44) & 28/101(27.72) & 0.000 \\
\hline Intensive care unit admission & $309 / 1690(18.28)$ & 191/1253(15.24) & 65/336(19.34) & $53 / 101(52.48)$ & 0.000 \\
\hline $\begin{array}{l}\text { Invasive mechanical } \\
\text { ventilation }\end{array}$ & $76 / 1690(4.50)$ & $36 / 1253(2.87)$ & $21 / 336(6.25)$ & 19/101(18.81) & 0.000 \\
\hline Composite endpoint $^{\mathrm{C}}$ & $314 / 1690(18.58)$ & $193 / 1253(15.40)$ & $66 / 336(19.64)$ & $55 / 101(54.46)$ & 0.000 \\
\hline $\begin{array}{l}\text { Time from symptom onset to } \\
\text { composite endpoint (days) }\end{array}$ & $21(13,33.5)$ & $24(15,38.5)$ & $19(13,27)$ & $16(11,30)$ & 0.015 \\
\hline \multicolumn{6}{|c|}{ Data are expressed as no./total no. (\%). } \\
\hline \multicolumn{6}{|c|}{ ap values were calculated using the chi-square test. } \\
\hline \multicolumn{6}{|c|}{$\begin{array}{l}\text { bRAS inhibitors included angiotensin-converting-enzyme inhibitors and angiotensin receptor blockers. RAS, renin- } \\
\text { angiotensin system. }\end{array}$} \\
\hline \multicolumn{6}{|c|}{$\begin{array}{l}{ }^{\mathrm{C}} \text { The composite endpoint comprised admission to the intensive care unit or mechanical ventilation or death, whichever } \\
\text { occurred first. }\end{array}$} \\
\hline \multicolumn{6}{|c|}{ COVID-19, coronavirus disease 2019; eGFR, estimated glomerular filtration rate } \\
\hline
\end{tabular}


Compared with the highest tertile of eGFR, the lower 2 tertiles were associated with more frequent use of HFNC, NMW, IMV, and CRRT. The use of antibiotics, glucocorticoids, RAS inhibitors, and diuretics was also significantly higher in the lower 2 tertiles than in the highest tertile. The frequency of complications was higher in the lower 2 tertiles than in the highest tertile. In addition to secondary infection, other complications were more common among patients in the lower 2 tertiles than among those in the highest tertile of eGFR; these included respiratory failure, ARDS, acute heart failure, acute cardiac injury, coagulation disorders, sepsis, shock, anemia, hypoproteinemia, electrolyte disturbances, and acidosis.

\section{Renal injury and in-hospital prognosis}

On admission, $4.7 \%$ and $13.5 \%$ patients had elevated SCr and BUN, respectively; $30.5 \%$ patients had hematuria, and relatively fewer patients (19.1\%) had proteinuria (Table 2 ). Only $5.3 \%$ and $1.9 \%$ patients had $2+-3+$ hematuria and proteinuria, respectively. Compared with patients in the highest tertile of eGFR, patients in the lower 2 tertiles had elevated renal dysfunction indicators (SCr and BUN). In addition, hematuria and proteinuria at presentation were more common in the lower 2 eGFR tertile than in the highest tertile (Table 2). During hospitalization, the peak SCr increased gradually with decreasing eGFR (Table 2). The incidence of AKI in the entire cohort was $6.7 \%$ according to KDIGO criteria (Table 3). Stage 1 AKI was present in $61.4 \%$ of the patients with AKI, while stages 2 and 3 were reached in $22.8 \%$ and $15.8 \%$ patients, respectively. The incidence of in-hospital AKI was higher in the lower 2 eGFR tertiles than in the highest tertile (Table 3 ).

A primary composite endpoint event occurred in 314 patients (18.6\%), including $18.3 \%$ who were admitted to the ICU, $4.5 \%$ who underwent IMV, and $4.9 \%$ who died (Table 3). The median time from symptom onset to composite endpoint was 21 days (IQR, 13.0-33.5 days). Compared with those in the highest tertile, significantly more patients in the lower 2 tertiles reached the composite endpoint ( $15.4 \%$ vs. $19.6 \%$ vs. $54.5 \%$; $P=0.000$; Table 3$)$. The rates of death $(2.4 \%$ vs. $7.4 \%$ vs. $27.7 \%$; $P=0.000)$, ICU admission ( $15.2 \%$ vs. $19.3 \%$ vs. $52.5 \% ; P=0.000)$, and IMV ( $2.9 \%$ vs. $6.3 \%$ vs. $18.8 \% ; P=0.000)$ also increased with decreasing eGFR. Compared to patients in the highest eGFR tertile, those in the lower 2 tertiles had shorter disease durations (from symptom onset to composite endpoint). Kaplan-Meier survival analysis revealed that survival until the composite endpoint was significantly lower in the lower 2 tertiles than in the highest tertile of eGFR $(P=0.000$, Fig. 1$)$. Patients with AKI had significantly escalated risks of reaching the composite endpoint compared with those without $A K I(P=0.000$, Fig. 2).

Two models of multivariate analysis were calculated. The first (Model 1) was designed to address whether eGFR predicted outcomes independently of disease severity, preexisting disorders, and baseline clinical data. The second model (Model 2) was designed to address whether eGFR was an independent predictor of outcomes even when follow-up data during hospitalization were taken into account; this model included disease severity, preexisting disorders, and baseline and follow-up data. Model 1 showed a significantly higher risk of the composite endpoint in the lowest eGFR tertile than in the higher 2 tertiles (reference: highest tertile, $\geq 90 \mathrm{~mL} / \mathrm{min} / 1.73 \mathrm{~m}^{2}$; middle tertile, $60-89 \mathrm{~mL} / \mathrm{min} / 1.73 \mathrm{~m}^{2}: \mathrm{HR}=1.538,95 \% \mathrm{Cl}: 0.978-2.421, \mathrm{P}=0.063 ;$ and lowest tertile, $<60 \mathrm{~mL} / \mathrm{min} / 1.73 \mathrm{~m}^{2}: \mathrm{HR}=1.953,95 \% \mathrm{Cl}: 1.174-3.250, \mathrm{P}=0.01$; Table 4). Model 2 showed that relative to the patients in the highest tertile, the HR $(95 \% \mathrm{Cl})$ was $1.535(0.976-2.413)(P=0.064)$ in the middle tertile and 1.942 (1.1683.230) $(P=0.011)$ in the lowest tertile. In both models, AKI was an independent risk factor for the composite endpoint, with a high HR of $1.724(95 \% \mathrm{Cl}: 1.070-2.778)(P=0.025)$ in Model 1 and an HR of $1.650(95 \% \mathrm{Cl}: 1.027-2.652)(P=0.039)$ in Model 2 (Table 4). The results of the unadjusted analysis are presented in Table 4. Overall, the findings of the unadjusted and adjusted analyses were not materially altered. 
Table 4

Multivariate Cox regression analysis of associations of baseline eGFR an1d in-hospital AKI with the c6omposite endpoint in patients with COVID-19

\begin{tabular}{|c|c|c|c|c|c|c|}
\hline \multirow[t]{2}{*}{ Variable } & \multirow{2}{*}{$\begin{array}{l}\text { Unadjusted } \\
\text { HR (95\% Cl) }\end{array}$} & \multirow{2}{*}{$\begin{array}{l}P \text { - } \\
\text { value }\end{array}$} & \multicolumn{4}{|c|}{ Adjusted HR (95\% Cl) } \\
\hline & & & Model $1^{a}$ & $\begin{array}{l}\mathrm{P}- \\
\text { value }\end{array}$ & Model $2^{b}$ & P-value \\
\hline \multicolumn{7}{|c|}{$\begin{array}{l}\text { eGFR, } \mathrm{ml} / \mathrm{min} / 1.73 \\
\mathrm{~m}^{2}\end{array}$} \\
\hline$\geq 90$ & 1.0(Ref) & & 1.0(Ref) & & 1.0(Ref) & \\
\hline $60-89$ & $\begin{array}{l}2.066(1.478- \\
2.889)\end{array}$ & 0.000 & $\begin{array}{l}1.538(0.978- \\
2.421)\end{array}$ & 0.063 & $\begin{array}{l}1.535(0.976- \\
2.413)\end{array}$ & 0.064 \\
\hline$₫ 60$ & $\begin{array}{l}5.595(3.938- \\
7.951)\end{array}$ & 0.000 & $\begin{array}{l}1.953(1.174- \\
3.250)\end{array}$ & 0.010 & $\begin{array}{l}1.942(1.168- \\
3.230)\end{array}$ & 0.011 \\
\hline \multicolumn{7}{|l|}{ AKI } \\
\hline without AKI & 1.0(Ref) & & 1.0(Ref) & & 1.0(Ref) & \\
\hline With AKI & $\begin{array}{l}5.263(3.831- \\
7.246)\end{array}$ & 0.000 & $\begin{array}{l}1.724(1.070- \\
2.778)\end{array}$ & 0.025 & $\begin{array}{l}1.650(1.027- \\
2.652)\end{array}$ & 0.039 \\
\hline \multicolumn{7}{|c|}{$\begin{array}{l}\text { a Model 1: adjusted for disease severity, hypertension, diabetes, coronary artery heart disease, cerebrovascular disease, } \\
\text { leukocyte count, lymphocyte count, CRP, IL-6, and procalcitonin }\end{array}$} \\
\hline \multicolumn{7}{|c|}{$\begin{array}{l}\text { bModel 2: adjusted for disease severity, hypertension, diabetes, coronary artery heart disease, cerebrovascular disease, } \\
\text { leukocyte count, lymphocyte count, CRP, IL-6, procalcitonin, and any complication }\end{array}$} \\
\hline \multicolumn{7}{|c|}{$\begin{array}{l}\text { Composite endpoint comprised admission to the intensive care unit or invasive ventilation or death, whichever occurred } \\
\text { first. }\end{array}$} \\
\hline
\end{tabular}

\section{Discussion}

In this multi-hospital observational study, we found that renal function was closely related to the prognosis of hospitalized COVID-19 patients. More than 25\% of the patients had impaired renal function, with an eGFR lower than $90 \mathrm{~mL} / \mathrm{min} / 1.73 \mathrm{~m}$. Patients with abnormal eGFR $\left(<90 \mathrm{~mL} / \mathrm{min} / 1.73 \mathrm{~m}^{2}\right)$ are more susceptible to severe COVID-19 infection. Although patients with impaired renal function received more intensive oxygen therapy, continuous blood purification, and glucocorticoid treatment, their prognosis was unsatisfactory, with a higher incidence of the composite endpoint (ICU admission, IMV, or death) and complications (AKI, respiratory failure, ARDS, acute cardiac injury, coagulation disorders, sepsis, shock, anemia, hypoproteinemia, electrolyte disturbances, and acidosis). Multivariate regression analysis showed that both eGFR $<60$ $\mathrm{mL} / \mathrm{min} / 1.73 \mathrm{~m}^{2}$ on admission and AKI occurrence during hospitalization were independent risk factors for poor in-hospital prognosis.

As the best overall index of kidney function, eGFR is the only criterion for staging CKD and determining long-term renal outcomes $^{12}$. A study of a multicenter registry found that $30 \%$ of COVID-19 patients had kidney dysfunction upon admission (eGFR $<60 \mathrm{~mL} / \mathrm{min} / 1.73 \mathrm{~m}^{2}$ ), which was associated with higher in-hospital mortality ${ }^{13}$. Our study showed that the incidence of complications, the composite endpoint $(15.4 \%$ vs. $19.6 \%$ vs. $54.5 \%, P=0.000)$, and mortality $(2.4 \%$ vs. $7.4 \%$ vs. $27.7 \%, P=$ 0.000 ) increased gradually with decreasing eGFR. The risk of reaching the composite endpoint among patients in the middle and lowest eGFR tertiles was 2.1 and 5.6 times, respectively, of the risk among those in the highest tertile $(P=0.000)$. This suggests that patients with renal function impairment (eGFR $<90 \mathrm{~mL} / \mathrm{min} / 1.73 \mathrm{~m}^{2}$ ) on admission had significantly worse inhospital outcomes, although the significance was lost in the middle tertile group (eGFR: $60-89 \mathrm{~mL} / \mathrm{min} / 1.73 \mathrm{~m}^{2}$ ) after 
adjustments for baseline and follow-up clinical data using the Cox regression model. Our results indicated that patients with an eGFR of $60-89 \mathrm{~mL} / \mathrm{min} / 1.73 \mathrm{~m}^{2}$ had already escalated risks of poor prognosis, emphasizing the need for early and continuous monitoring of renal function.

The prevalence of CKD in China is $10.8 \%^{14}$. However, in our study, only $2.6 \%$ of patients with COVID-19 had a history of CKD. We also found that $19.1 \%$ and $30.5 \%$ patients had proteinuria and hematuria, respectively. Thus, CKD may be underestimated in these patients on admission. Fever is the most common clinical symptom in COVID-19 patients, with a high incidence of $87.9-94 \%{ }^{15,16}$. An interesting finding in our study was that patients with kidney injury, especially those with GFR $<60$ $\mathrm{mL} / \mathrm{min} / 1.73 \mathrm{~m}^{2}$, were less likely to develop fever, with an incidence of less than $50 \%$. Despite a low incidence of clinical symptoms, patients with eGFR $<90 \mathrm{~mL} / \mathrm{min} / 1.73 \mathrm{~m}^{2}$ had a higher incidence of complications, the composite endpoint, and mortality. This may be due to low immunity (reduced lymphocyte count) ${ }^{17,18}$ coagulation disorders (decreased platelet count, prolonged prothrombin time, and increased d-dimer) ${ }^{19,20}$, poor nutrition (anemia and hypoproteinemia), and persistent inflammatory states (high procalcitonin, interleukin-6, and C-reactive protein) ${ }^{21}$ in patients with impaired renal function (eGFR< $90 \mathrm{~mL} / \mathrm{min} / 1.73 \mathrm{~m}^{2}$ ). Therefore, these patients should receive immunotherapy, nutrition support, and anti-inflammatory and anticoagulant therapy. Some novel treatments have good therapeutic effects in COVID-19, such as tocilizumab ${ }^{21}$, umbilical cord mesenchymal stem cells ${ }^{11}$, and convalescent plasma ${ }^{22}$. However, we could not evaluate the effects of these treatments in patients with different renal function due to the small number of cases.

Acute kidney injury (AKI), as a common complication of COVID-19, is usually related to disease mortality ${ }^{23}$. A postmortem patient series found significant acute tubular injury in all patients who had died of COVID-1924. The mechanisms of kidney injury in SARS-CoV-2 infection include direct viral injury via the angiotensin-converting enzyme 2 receptor, which is highly expressed in the kidneys ${ }^{25}$, an imbalanced renin-angiotensin-aldosterone system ${ }^{26,27}$ and release of proinflammatory cytokines elicited by the viral infection and microvascular thrombosis ${ }^{28}$. We found that patients with eGFR $<60 \mathrm{~mL} / \mathrm{min} / 1.73 \mathrm{~m}{ }^{2}$ were more likely to develop AKI during hospitalization, with an incidence of $17 \%$. Furthermore, $10 \%$ of patients with eGFR $<60$ $\mathrm{mL} / \mathrm{min} / 1.73 \mathrm{~m}^{2}$ received CRRT. A study in New York has reported a high incidence of AKI (37-46\%) among COVID-19 patients ${ }^{23}$ and $19 \%$ of patients with AKI required dialysis, and half of them died in the hospital. We found that AKI was an independent risk factor in patients with COVID-19 after adjustments. Therefore, regular monitoring of renal function and timely diagnosis of AKI are conducive to the treatment of COVID-19 patients.

Our study has some limitations. First, the incidence of CKD and AKI may be underestimated in some patients due to the lack of baseline medical records, late admission, and lack of renal function examination after admission. Second, a small proportion of patients were still in the hospital, and their outcomes were unknown at the time of the data cutoff, which might lead to the underestimation of the endpoint events. Third, due to different diagnostic paradigms in different hospitals, not all laboratory tests were performed in all patients, which led to some missing data. Last but not the least, there was no direct evidence of renal damage caused by the virus in the urine or kidney tissue.

In conclusion, impaired renal function on admission and the occurrence of AKI during hospitalization are independent predictors of poor prognosis among hospitalized COVID-19 patients. Therefore, early and continuous monitoring of renal function and early diagnosis of AKI are necessary interventions to predict and prevent the progression of COVID-19.

\section{Methods}

\section{Study design and participants}

This retrospective, multicenter study included 3 cohorts of 1851 adult in-patients ( $\geq 18$ years) with confirmed COVID-19 pneumonia who were hospitalized in 3 hospitals that are designated care centers for patients with emerging infectious diseases in Wuhan (Tongji Taikang Hospital, Huo Shen Shan Hospital, and Renmin Hospital of Wuhan University) between February 3, 2020 and April 10, 2020.

Page $13 / 18$ 
All patients were diagnosed with COVID-19 pneumonia according to $\mathrm{WHO}$ interim guidance ${ }^{29}$. Confirmed cases denoted patients with positive findings on high-throughput sequencing or real-time reverse-transcription polymerase chain reaction assays of nasal and pharyngeal swab specimens ${ }^{5}$. Patients without renal function tests were excluded. Participants were followed up until discharge or in-hospital demise.

This study was approved by the Research Ethics Commission of each participating site.

All methods were carried out in accordance with relevant guidelines and regulations.

Written informed consent was waived by the Ethics Commissions of the designated hospitals for emerging infectious disease.

\section{Data collection and definitions}

Demographic characteristics, clinical data (symptoms, comorbidities, treatments, complications, and outcomes data), laboratory findings, and chest computed tomography (CT) findings were retrieved from electronic medical records by 4 investigators (F.X., Y.L., X.D.L, Y.Y., and Y.L). The eGFR was calculated using the Chronic Kidney Disease Epidemiology Collaboration Eq. $3^{0}$. AKI was defined as an increase in SCr of $\geq 26.5 \mu \mathrm{mol} / \mathrm{L}$ within $48 \mathrm{~h}$ or a $50 \%$ increase in SCr from the baseline within 7 days, according to the Kidney Disease - Improving Global Outcomes (KDIGO) criteria ${ }^{31}$. Baseline SCr was defined as the SCr value on admission. The date of AKI onset was defined as the earliest day on which a SCr change meeting the KDIGO criteria was recorded. The stage of AKI was determined using the peak SCr level after AKI detection, with increases of 1.5-1.9, 2.0-2.9, and $\geq 3$ times the baseline value being defined as AKI stages 1, 2, and 3, respectively. The severity of COVID-19 was defined according to the Interim Guidelines for COVID-19 of China (6th edition) provided by the National Health Commission of China. Acute respiratory failure was defined as a decrease in oxygen saturation (<92\%) while breathing room air with severe respiratory distress or hypoxemia (partial oxygen pressure $<60 \mathrm{~mm} \mathrm{Hg}$ ) and/or requirement of invasive/noninvasive mechanical ventilation. Shock and acute respiratory distress syndrome (ARDS) were defined in accordance with WHO interim guidance ${ }^{29}$. Acute cardiac injury was diagnosed if serum levels of cardiac biomarkers (e.g., highsensitivity cardiac troponin I) were above the 99th percentile upper reference limit, or if new abnormalities were observed on electrocardiography and echocardiography ${ }^{32}$. Acute heart failure was defined as the clinical syndrome characterized by typical symptoms (e.g., breathlessness, ankle swelling, and fatigue) that may be accompanied by signs (e.g., elevated jugular venous pressure, pulmonary crackles, and peripheral edema) caused by a structural and/or functional cardiac abnormality ${ }^{33}$. Sepsis and septic shock were defined according to the 2016 Third International Consensus Definition for Sepsis and Septic Shock ${ }^{5}$. Secondary infection was diagnosed when patients showed clinical symptoms or signs of pneumonia or bacteremia, and a positive culture of a new pathogen was obtained from lower respiratory tract specimens (qualified sputum, endotracheal aspirate, or bronchoalveolar lavage fluid) or blood samples after admission ${ }^{5}$. Anemia was defined as a hemoglobin level of < $120 \mathrm{~g} / \mathrm{L}$ for male patients or $<110 \mathrm{~g} / \mathrm{L}$ for female patients. Hypoproteinemia was defined as a blood albumin level of $<35 \mathrm{~g} / \mathrm{L}$.

\section{Statistical analysis}

Categorical variables were expressed as counts and percentages, and continuous variables were expressed as medians with interquartile ranges (IQRs). The Kruskal-Wallis test was applied to continuous variables, and the chi-square test and Fisher exact test were used for categorical variables, as appropriate. The composite endpoint was ICU admission, invasive ventilation, or death, whichever occurred first. Survival curves for the composite endpoint were derived using the Kaplan-Meier method, and differences between curves were analyzed using the log-rank test. Multivariate Cox regression models were used to test the associations of baseline eGFR and in-hospital AKI with the composite endpoint during hospitalization. The results are presented as hazard ratios (HRs) with $95 \%$ confidence intervals (95\% Cls) and P values. All statistical analyses were performed using SPSS 22.0 (IBM, Armonk, NY, USA). For all analyses, P $\mathbb{0} 0.05$ (two-tailed) was considered significant.

\section{Declarations}

\section{Acknowledgements}

This work was supported by the National Key Research and Development Project (No. 2018YFC1312705), National Natural Science Foundation of China (No. 81770731), and Chongqing Municipal Natural Science Foundation (No. cstc2020jcyj-

Page $14 / 18$ 
msxmX0013).

\section{Authors' contributions}

H.D. and J.W. conceived and designed the study. Y.L., F.X., Y.Y., X.L. and Y.L. collected the data. K.C. and Y.L. performed the data analysis. Y.H. checked the data. H.D. prepared the tables and figures. H.D. and K.C. edited the manuscript. All authors read and approved the final manuscript.

\section{Competing financial interests}

The authors declare no competing financial interests.

\section{References}

1. Zhu, N. et al. A Novel Coronavirus from Patients with Pneumonia in China, 2019. N Engl J Med. 382, 727-733 (2020).

2. Puelles, V. G. et al. Multiorgan and Renal Tropism of SARS-CoV-2. N Engl J Med. 383, 590-592 (2020).

3. Belhadjer, Z. et al. Acute heart failure in multisystem inflammatory syndrome in children (MIS-C) in the context of global SARS-CoV-2 pandemic. Circulation. 142,, 429-436 (2020).

4. Cheng, Y. et al. Kidney disease is associated with in-hospital death of patients with COVID-19. Kidney Int. 97, 829-838 (2020).

5. Huang, C. et al. Clinical features of patients infected with 2019 novel coronavirus in Wuhan, China. Lancet. 395, 497-506 (2020).

6. Wang, D. et al. Clinical Characteristics of 138 Hospitalized Patients With 2019 Novel Coronavirus-Infected Pneumonia in Wuhan, China. JAMA. 323, 1061-1069 (2020).

7. Guan, W. J. et al. Clinical Characteristics of Coronavirus Disease 2019 in China. N Engl J Med. 382, 1708-1720 (2020).

8. Yang, X. et al. Clinical course and outcomes of critically ill patients with SARS-CoV-2 pneumonia in Wuhan, China: a singlecentered, retrospective, observational study. Lancet Respir Med. 8, 475-481 (2020).

9. Gabarre, P. et al. Acute kidney injury in critically ill patients with COVID-19. Intensive Care Med. 46, 1339-1348 (2020).

10. Wang, L. et al. Coronavirus Disease 19 Infection Does Not Result in Acute Kidney Injury: An Analysis of 116 Hospitalized Patients from Wuhan, China. American journal of nephrology. Am J Nephrol. 51, 343-348 (2020).

11. Meng, F. et al. Human umbilical cord-derived mesenchymal stem cell therapy in patients with COVID-19: a phase 1 clinical trial. Signal Transduct Target Ther. 5, 172 (2020).

12. Choi, H. Y. et al. The Power of Renal Function Estimation Equations for Predicting Long-Term Kidney Graft Survival: A Retrospective Comparison of the Chronic Kidney Disease Epidemiology Collaboration and the Modification of Diet in Renal Disease Study Equations. Medicine (Baltimore). 95, e2682 (2016).

13. Uribarri, A. et al. Impact of renal function on admission in COVID-19 patients: an analysis of the international HOPE COVID19 (Health Outcome Predictive Evaluation for COVID 19) Registry. J Nephrol. 33, 737-745 (2020).

14. Zhang, L. et al. Prevalence of chronic kidney disease in China: a cross-sectional survey. Lancet. 379, 815-822 (2012).

15. Guan, W. J. et al. Clinical Characteristics of Coronavirus Disease 2019 in China. N Engl J Med. 382, 1708-1720 (2020).

16. Zhou, F. et al. Clinical course and risk factors for mortality of adult inpatients with COVID-19 in Wuhan, China: a retrospective cohort study. Lancet. 395, 1054-1062 (2020).

17. Tan, L. et al. Lymphopenia predicts disease severity of COVID-19: a descriptive and predictive study. Signal Transduct Target Ther. 5, 33 (2020).

18. Huang, I. \& Pranata, R. Lymphopenia in severe coronavirus disease-2019 (COVID-19): systematic review and metaanalysis. J Intensive Care. 8, 36 (2020).

19. Ramlall, V. et al. Immune complement and coagulation dysfunction in adverse outcomes of SARS-CoV-2 infection. Nat Med. 26, 1609-1615 (2020). 
20. Ackermann, M. et al. Pulmonary Vascular Endothelialitis, Thrombosis, and Angiogenesis in Covid-19. N Engl J Med. 383, 120-128 (2020).

21. Betjes, M. G. Immune cell dysfunction and inflammation in end-stage renal disease. Nat Rev Nephrol. 9, 255-265 (2013).

22. Li, L. et al. Effect of Convalescent Plasma Therapy on Time to Clinical Improvement in Patients With Severe and Lifethreatening COVID-19: A Randomized Clinical Trial. JAMA. 324, 460-470 (2020).

23. Chan, L. et al. AKI in Hospitalized Patients with COVID-19. J Am Soc Nephrol. 32, 151-160 (2021).

24. Su, H. et al. Renal histopathological analysis of 26 postmortem findings of patients with COVID-19 in China. Kidney Int. 98 , 219-227 (2020).

25. Gheblawi, M. et al. Angiotensin-Converting Enzyme 2: SARS-CoV-2 Receptor and Regulator of the Renin-Angiotensin System: Celebrating the 20th Anniversary of the Discovery of ACE2. Circ Res. 126, 1456-1474 (2020).

26. Cabbab, I. L. N. \& Manalo, R. V. M. Anti-Inflammatory Drugs and the Renin-Angiotensin-Aldosterone System: Current Knowledge and Potential Effects on Early SARS-CoV-2 Infection. Virus Res. 291, 198190 (2021).

27. Dudoignon, E. et al. Activation of the renin-angiotensin-aldosterone system is associated with Acute Kidney Injury in COVID-19. Anaesth Crit Care Pain Med. 39, 453-455 (2020).

28. Nicolai, L. et al. Immunothrombotic Dysregulation in COVID-19 Pneumonia Is Associated With Respiratory Failure and Coagulopathy. Circulation. 142, 1176-1189 (2020).

29. WHO: Clinical management of severe acute respiratory infection when novel coronavirus (2019-nCoV) infection is suspected: interim guidance, 28 January 2020, Available at: https://apps.who.int/iris/handle/10665/330893.

30. Levey, A. S. et al. A new equation to estimate glomerular filtration rate. Ann Intern Med. 150, 604-612 (2009).

31. Kellum, J. A. et al. Kidney disease: Improving global outcomes (KDIGO) acute kidney injury work group. KDIGO clinical practice guideline for acute kidney injury. Kidney International Supplements. 2, 1-138 (2012).

32. Shi, S. et al. Association of Cardiac Injury With Mortality in Hospitalized Patients With COVID-19 in Wuhan, China. JAMA Cardiol. 5, 802-810 (2020).

33. Ponikowski, P. et al. 2016 ESC Guidelines for the diagnosis and treatment of acute and chronic heart failure: The Task Force for the diagnosis and treatment of acute and chronic heart failure of the European Society of Cardiology (ESC)Developed with the special contribution of the Heart Failure Association (HFA) of the ESC. Eur Heart J. 37, 21292200 (2016).

\section{Figures}




\section{- eGFR $<60$ \\ $-60 \leqslant \mathrm{eGFR} \leqslant 89$ \\ - eGFR $\geqslant 90$}

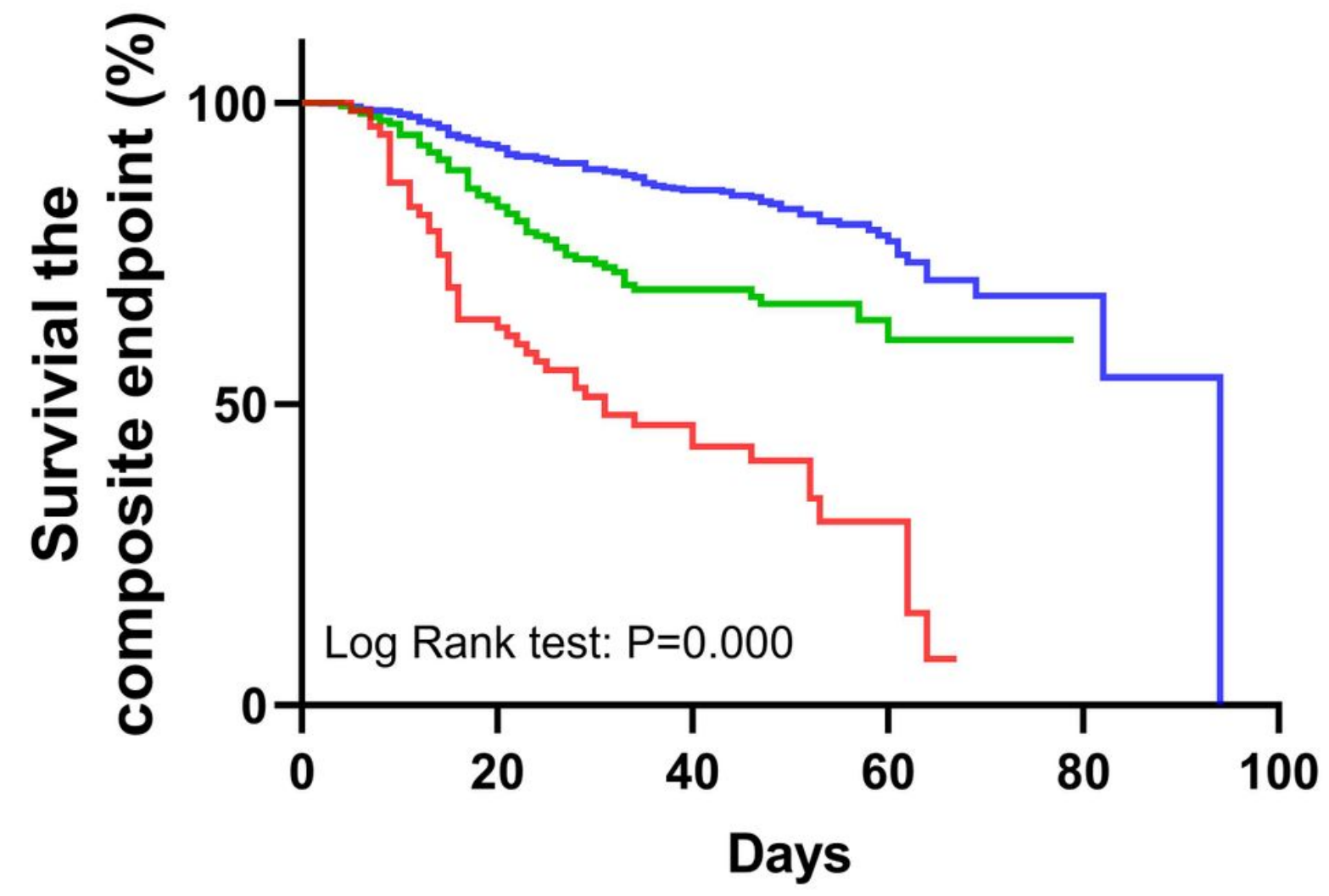

Figure 1

Kaplan-Meier survival curves for the composite endpoint among patients with COVID-19 stratified according to eGFR COVID19, coronavirus disease 2019; eGFR, estimated glomerular filtration rate 


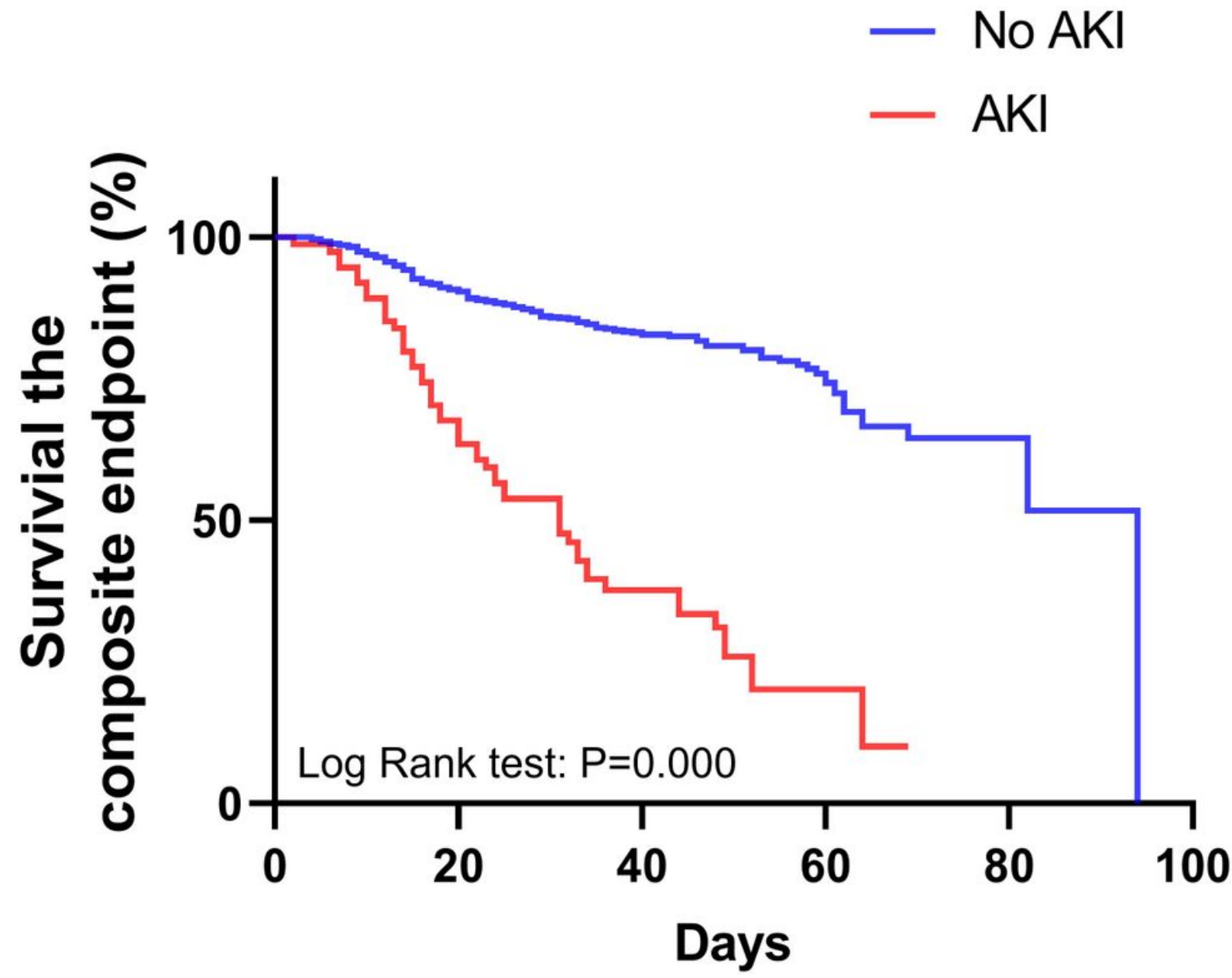

Figure 2

Kaplan-Meier survival curves for the composite endpoint among COVID-19 patients with and without AKI COVID-19, coronavirus disease 2019; AKI, acute kidney injury 